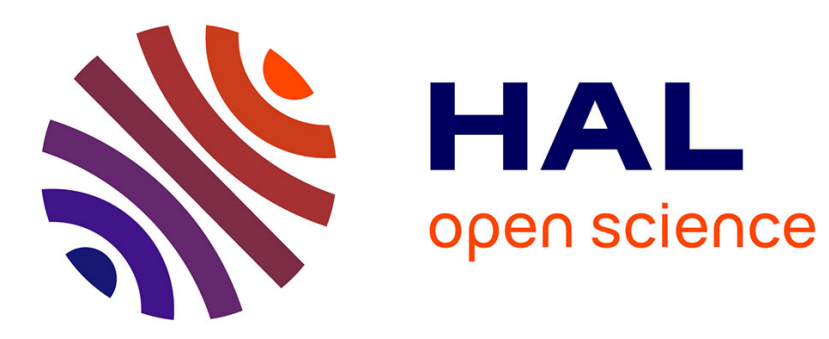

\title{
Alternative method for Hamilton-Jacobi PDEs in image processing
}

Aurélie Lagoutte, Hadrien Salat, Corinne Vachier

\section{To cite this version:}

Aurélie Lagoutte, Hadrien Salat, Corinne Vachier. Alternative method for Hamilton-Jacobi PDEs in image processing. Image Processing: Algorithms and Systems IX, Jan 2011, San Francisco, CA, United States. pp.787010, 10.1117/12.872591 . hal-01508512

\section{HAL Id: hal-01508512 \\ https://hal.science/hal-01508512}

Submitted on 14 Apr 2017

HAL is a multi-disciplinary open access archive for the deposit and dissemination of scientific research documents, whether they are published or not. The documents may come from teaching and research institutions in France or abroad, or from public or private research centers.
L'archive ouverte pluridisciplinaire HAL, est destinée au dépôt et à la diffusion de documents scientifiques de niveau recherche, publiés ou non, émanant des établissements d'enseignement et de recherche français ou étrangers, des laboratoires publics ou privés. 


\title{
Alternative method for Hamilton-Jacobi PDEs in image processing
}

\author{
A. Lagoutte ${ }^{a}$, H. Salat ${ }^{a}$, and C. Vachier ${ }^{a}$ \\ ${ }^{a}$ ENS Cachan, CNRS, PRES UniverSud, 61 Av. President Wilson, F-94230 Cachan, FRANCE.
}

\begin{abstract}
Multiscale signal analysis has been used since the early 1990s as a powerful tool for image processing, notably in the linear case. However, nonlinear PDEs and associated nonlinear operators have advantages over linear operators, notably preserving important features such as edges in images. In this paper, we focus on nonlinear Hamilton-Jacobi PDEs defined with adaptive speeds or, alternatively, on adaptive morphological fiters also called semi-flat morphological operators. Semi-flat morphology were instroduced by H. Heijmans and studied only in the case where the speed (or equivalently the filtering parameter) is a decreasing function of the luminance. It is proposed to extend the definition suggested by H. Heijmans in the case of non decreasing speeds. We also prove that a central property for defining morphological filters, that is the adjunction property, is preserved while dealing with our extended definitions. Finally experimental applications are presented on actual images, including connection of thin lines by semi-flat dilations and image filtering by semi-flat openings.
\end{abstract}

Keywords: Mathematical Morphology, Nonlinear PDEs, Adaptive Filtering.

\section{INTRODUCTION}

In image processing important problems such as image smoothing, detecting events (e.g. edges, peaks) or removing noises from an image often require analysing a signal at multiple spatial or intensity scales. Until now, most of these problems have been addressed using linear filters or PDE. ${ }^{1}$ There is however, a variety of nonlinear filters such as openings and closings that could be used providing some advantages over linear filters, notably leaving the edges unchanged.

Most nonlinear filters are based on a PDE whose role is to link scale shifting to spatial shifting. ${ }^{2-4}$ For this purpose, Hamilton-Jacobi PDEs are a very productive tool. They are nonlinear differential equations of type

$$
\frac{\partial F}{\partial t}=V(F(x, y, t)) \cdot\|\nabla F\|
$$

with $V$ a function depending on the solution. Because of this dependence, $V$ gives the PDE an adaptive characteristic.

It has been shown that solving the case $V$ constant, namely the linear case of Hamilton-Jacobi PDEs, has the same effect as applying to a function some morphological operators, namely operators over a complete lattice. ${ }^{1}$ A complete lattice is a set fitted with a total or partial order, whose every subset has an inferior and a superior bounds. The complete lattice is mainly used afterwards applied to the set of grey-scale images, which means the set of functions from a bounded subset of $\mathbb{R}^{2}$ (or $\mathbb{Z}^{2}$ ) in a bounded subset of $\mathbb{R}$ (or $\mathbb{Z}$ ), fitted with the classical order on functions. ${ }^{5}$

The case $V=1$ and $V=-1$ match respectively the dilatation and erosion operators defined for numerical functions. ${ }^{2,3}$ As illustrated in Fig 1, if $X \subseteq \mathbb{R}^{2}$ and $B$ is the unit ball, then the dilation of a set $X$ by a ball $B$ is obtained by taking the union of all points included in the ball placed successively in such a way that its center covers all elements in $X$. The erosion of a set $X$ by $B$ is obtained by keeping the points $x$ of $X$ such that the unit ball centered in $x$ is entirely included in $X$. Formal definitions are:

(Send correspondence to C. Vachier)

E-mail: Corinne.Vachier@cmla.ens-cachan.fr, Telephone: +33 (0)1 47405941 


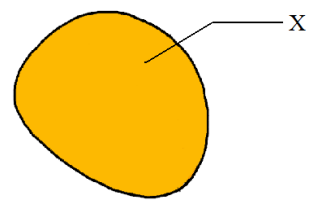

(a) $X$

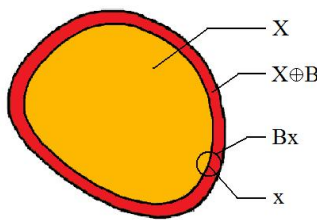

(b) Dilation of $X$ by $B$

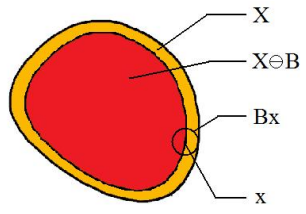

(c) Erosion of $X$ by $B$

Figure 1. Erosion and dilation in $\mathbb{R}^{2}$

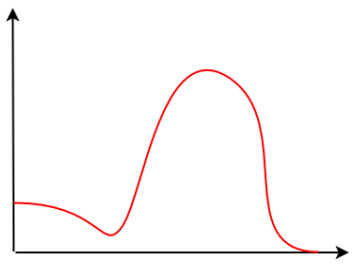

(a) $y=F(x)$

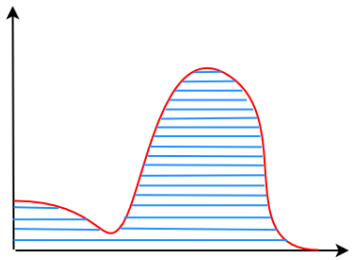

(b) Level sets of $F$

Figure 2. Level sets of $F$

- Dilation: $X \oplus B=\bigcup_{b \in B} X_{b}$

- Erosion: $X \ominus B=\bigcap_{b \in B} X_{-b}$

Where $X_{b}$ is the set $X$ translated by the vector $b: X_{b}=\{x+b \mid x \in X\}$

Now, dilations and erosions are defined for functions by the level set extension paradigm. ${ }^{3,5,6}$ This point will be detailed in the following section of this paper.

More elaborated useful cases of Hamilton-Jacobi are presented in. ${ }^{7-11}$ Relying on all these existing works, one can think solving Hamilton-Jacobi PDEs in the general nonlinear case also has the effect of other morphological operators, that can be used in image processing.

In this paper, the purpose of the first part is to introduce flat and classical semi-flat morphology, respectively operating with a constant and decreasing family of morphological operators. The second part is devoted to extending these tools in the general case of any family of operators, and showing that this transformation is adjunction-preserving. In the last part, the results of experimental applications of morphological filters to images are gathered.

\section{FLAT AND SEMI-FLAT MORPHOLOGIES}

\subsection{Flat morphology}

Flat morphology ${ }^{5,6}$ consists in applying the same morphological operator to an indexed family of sets, for example the level sets of a function or the grey scales of an image. The morphological operator used will mostly be a dilation or an erosion.

To begin with, the notion of level set of a function should be introduced. Let $\mathcal{L}$ be a complete lattice and $F: E \rightarrow \mathcal{L}$ a function. There are two ways to define the level sets of $F:$

$$
X(F, s)=\{x \in E \mid F(x) \geq s\} \quad \text { or } \quad Y(F, s)=\{x \in E \mid F(x)>s\}
$$

$X(F, s)$ and $Y(F, s)$ define the part of $E$ for which the function is above a certain value $s$. Their difference lies in whether the points of the function itself should be included or not. See Fig.2 for an illustration of a function and its level sets. 
For a given function $F$, we now apply a constant set dilation (or erosion) to its level sets to create a dilated (or eroded) function from $F$. The same can be done with a grey scale image by applying a constant set dilation (or erosion) to the sets formed by the level sets of the function $\left(\mathbb{R}^{2} \rightarrow \mathbb{R}_{+}\right.$or $\left.\mathbb{N}^{2} \rightarrow \mathbb{N}\right)$ representing the image.

A dilation $\delta$ applied to the function $F$ level sets is shown on Fig.3

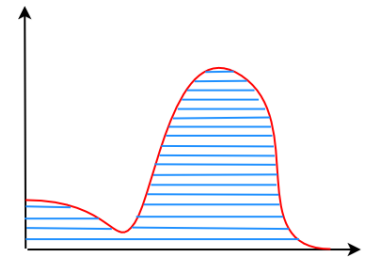

(a) Level sets of $F$

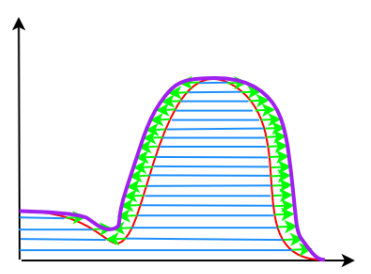

(b) Dilation of $F$

Figure 3. Dilation of $F$

If $\delta$ is a set dilation, then the operator $\Delta$ that transforms every function $F: x \mapsto F(x)$ into

$$
x \mapsto \sup \{s \in \mathcal{L} \mid x \in \delta X(F, s)\}
$$

also defines a dilation. Of course the same holds for erosions. When applying flat dilations or erosions to functions, the dilations or erosions being defined using balls of increasing size $t$, we obtain the same results as resolving the Hamilton-Jacobi PDE special cases of $V=1$ and $V=-1$ seen in the introduction. ${ }^{2,3}$

\subsection{Semi-flat morphology with an ordinary decreasing family of morphological operators}

Let $\mathcal{L}$ be a complete lattice and $F: E \rightarrow \mathcal{L}$ a function. Adaptive morphology is defined by Heijmans in ${ }^{6}$ for a decreasing family $\left(\psi_{s}\right)_{s \in \mathcal{L}}$ of increasing operators (for all $s \leq s^{\prime} \in \mathcal{L}, \psi_{s^{\prime}} \leq \psi_{s}$ and for all $s \in \mathcal{L}$, and $\psi_{s}$ is supposed to be an increasing operator. The semi-flat extension of the family $\left(\psi_{s}\right)_{s \in \mathcal{L}}$ consists in applying for each $s \in \mathcal{L}$ the operator $\psi_{s}$ to the level set $X(F, s)$ of $F$, then reconstructing the function whose level sets are $\psi_{s}(X(F, s)){ }^{6}$ The resulting function is defined by

$$
x \mapsto \sup \left\{s \in \mathcal{L} \mid x \in \psi_{s} X(F, s)\right\}
$$

We know for sure that we will be able to do this reconstruction because the images of stacked level sets by a decreasing family of operators are also stacked, which means that those images are still the level sets of a properly defined function.

We show in Fig.4 the transformation of $F$ by an adaptive morphology using a family $\left(\delta_{s}\right)_{s \in \mathcal{L}}$ such that $\delta_{s}$ is a dilation by a ball whose radius gets smaller as $s$ increases.

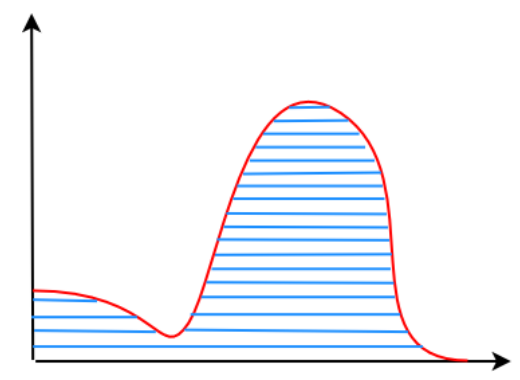

(a) Level sets of $F$

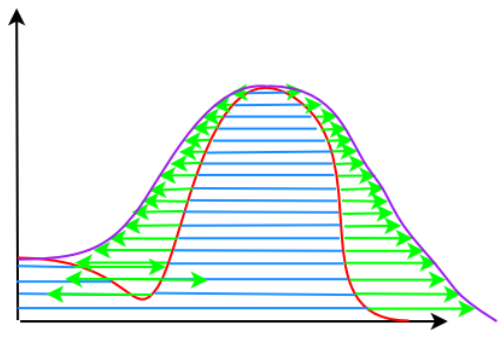

(b) adaptive dilation applied on $F$

Figure 4. The semi-flat extension paradigm 


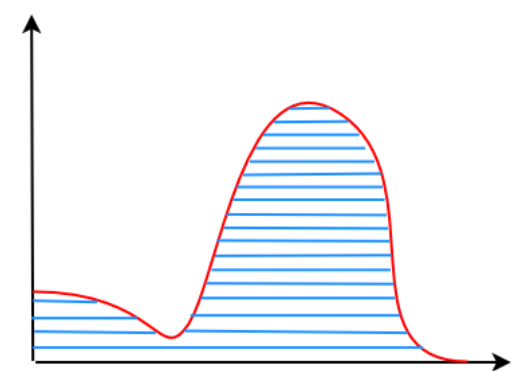

(a) Level sets of $F$

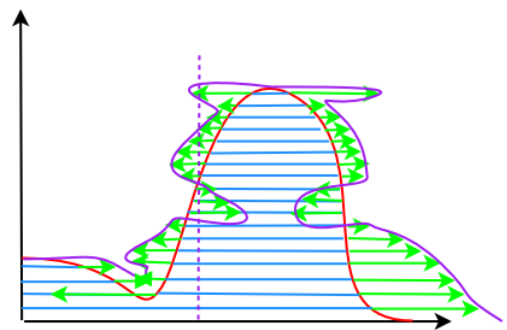

(b) Transformed level sets of $F$

Figure 5. The problem of the semi-flat extension in the case of a non decreasing family of set operators

\section{EXTENDED ADAPTIVE MORPHOLOGY}

\subsection{Extension of Heijmans'semi-flat morphology}

Here lies one of the main goals of this paper: extending the notion of adaptive morphology to any family $\left(\psi_{s}\right)_{s \in \mathcal{L}}$ of morphological operators. It implies as a first step that we should be able to apply the operator $\psi_{s}$ to $X(F, s)$ for each $s$ and still obtain a properly defined function. Unfortunately, as the family $\left(\psi_{s}\right)$ is not supposed to be decreasing any more, the transformed sets $\psi_{s}(X(F, s))$ may not remain stacked, as it is enhanced in Fig.5. Hence, the reconstruction of a function is not immediate.

Two solutions to this problem are graphically described on Fig.?? : sup-envelope and inf-envelope. Intuitively, the sup-envelope acts as water falling in a fountain, following the borders delimited by the extremities of the transformed level sets. The inf-envelope is inferred by the same idea applied to the opposite function $-F$. The formal definitions of these envelopes are the following ones: if $(Z(s))_{s \in \mathcal{L}}$ is a family of subsets of $E$, then

- the sup-envelope is defined by

$$
\bar{F}:\left\{\begin{array}{ccc}
E & \rightarrow & \mathcal{T} \\
x & \mapsto & \sup \{s \in \mathcal{L} \mid x \in Z(s)\}
\end{array}\right.
$$

- and the inf-envelope is defined by

$$
\underline{F}:\left\{\begin{array}{ccc}
E & \rightarrow & \mathcal{T} \\
x & \mapsto & \inf \{s \in \mathcal{L} \mid x \notin Z(s)\}
\end{array}\right.
$$

Now, for defining semi-flat extensions of families of set operators, one can set $Z(s)=\psi_{s}(X(F, s))$ or $Z(s)=$ $\psi_{s}(Y(F, s))$ in order to define transformed functions as inf- or sup-envelopes.

\subsection{Special case of adaptive dilations and erosions}

The semi-flat extension of families of set operators being defined for any family, let us prove now that some useful properties satisfied in the case of decreasing families are still true even if the family is not decreasing. We will focus on the adjunction property that plays a central role in mathematical morphology for defining morphological filters, that is, operators acting on images that are idempotent. ${ }^{5}$

Let $\mathcal{L}, \mathcal{M}$ be complete lattices, $\varepsilon$ an operator from $\mathcal{L}$ to $\mathcal{M}$, and $\delta$ an operator from $\mathcal{M}$ to $\mathcal{L}$. The pair $(\varepsilon, \delta)$ is called an adjunction between $\mathcal{L}$ and $\mathcal{M}$ if

$$
\forall X \in \mathcal{L}, \forall Y \in \mathcal{M}, \delta(X) \leq X \Leftrightarrow Y \leq \varepsilon(X)
$$

Adjunctions are narrowly linked to dilations and erosions. Indeed, if the pair $(\varepsilon, \delta)$ is an adjunction, then $\varepsilon$ must be an erosion and $\delta$ a dilation. But here dilations and erosions are to be understood in the broader sense of algebraic dilations and algebraic erosions. 
An algebraic dilation is any operator which commutes with union $\left(\delta\left(\bigvee_{i \in I} Y_{i}\right)=\bigvee_{i \in I} \delta\left(Y_{i}\right)\right)$ and an algebraic erosion is any operator which commutes with intersection $\left(\varepsilon\left(\bigwedge_{i \in I} Y_{i}\right)=\bigwedge_{i \in I} \varepsilon\left(Y_{i}\right)\right)$. Given an adjunction $(\varepsilon, \delta)$, the products $\varepsilon \delta$ and $\delta \varepsilon$ respectively define openings and closings.

Let $\left(\varepsilon_{s}, \delta_{s}\right)_{s \in \mathbb{R}}$ be a family of adjunctions on $\mathbb{R}^{2}$. Let us define $\underline{\Sigma}$ and $\bar{\Delta}$, two morphological operators such that for any function $F$ from a compact subset of $\mathbb{R}^{2}$ to $\mathbb{R}, \underline{\Sigma}(F)$ is the inf-envelope of the level sets of $F$ eroded by the family $\left(\varepsilon_{s}\right)_{s \in \mathcal{L}}$ and $\bar{\Delta}(F)$ is the sup-envelope of the level sets of $F$ dilated by the family $\left(\delta_{s}\right)_{s \in \mathcal{L}}$ :

$\Sigma:\left\{\begin{array}{l}\mathcal{C}\left(\mathbb{R}^{2}, \mathbb{R}\right) \rightarrow \mathcal{C}\left(\mathbb{R}^{2}, \mathbb{R}\right) \\ F \mapsto \Sigma(F): x \mapsto \wedge\left\{s \in \mathbb{R} \mid x \notin \varepsilon_{s} X(F, s)\right\}\end{array}\right.$
$\bar{\Delta}:\left\{\begin{array}{l}\mathcal{C}\left(\mathbb{R}^{2}, \mathbb{R}\right) \rightarrow \mathcal{C}\left(\mathbb{R}^{2}, \mathbb{R}\right) \\ F \mapsto \bar{\Delta}(F): x \mapsto \vee\left\{s \in \mathbb{R} \mid x \in \delta_{s} Y(F, s)\right\}\end{array}\right.$

Strict and large level sets in the definition of $\underline{\Sigma}$ and $\bar{\Delta}$ are chosen in a way that makes the following property true and $\mathcal{C}\left(\mathbb{R}^{2}, \mathbb{R}\right)$ is the subset formed by all functions of $\mathcal{F}\left(\mathbb{R}^{2}, \mathbb{R}\right)$ with compact support.

Theorem $(\underline{\Sigma}, \bar{\Delta})$ is an adjunction on $\mathcal{C}\left(\mathbb{R}^{2}, \mathbb{R}\right)$.

Proof Let us prove that

$$
\forall F, G \in \mathcal{C}\left(\mathbb{R}^{2}, \mathbb{R}\right), \bar{\Delta}(F) \leq G \Longleftrightarrow F \leq \underline{\Sigma}(G)
$$

\section{Forward}

Let $F, G \in \mathcal{C}\left(\mathbb{R}^{2}, \mathbb{R}\right)$.

$$
\begin{gathered}
\text { As } \forall y \in \mathbb{R}^{2}, \quad \bar{\Delta}(F)(y) \leq G(y), \\
\text { then } \vee\left\{s \mid y \in \delta_{s} Y(F, s)\right\} \leq G(y), \\
\text { so } \forall s \in \mathcal{T}, y \in \delta_{s} Y(F, s) \Longrightarrow s \leq G(y) . \\
\text { We deduce } \delta_{s} Y(F, s) \subseteq\{y \mid G(y) \geq s\}, \\
\text { and by adjunction } Y(F, s) \subseteq \varepsilon_{s}(\{y \mid G(y) \geq s\}), \\
\text { which means } Y(F, s) \subseteq \varepsilon_{s}(X(G, s)) .
\end{gathered}
$$

Then, as we have the following relation on the complementaries:

$$
\varepsilon_{s}(X(G, s))^{c} \subseteq Y(F, s)^{c}
$$

We deduce for the inferior bounds that $\forall x$,

$$
\underbrace{\wedge\left\{s \mid x \in \varepsilon_{s}(X(G, s))^{c}\right\}}_{\underline{\Sigma}(G)(x)} \geq \underbrace{\wedge\left\{s \mid x \in Y(F, s)^{c}\right\}}_{F(x)}
$$

Eventually, we obtain:

$$
\underline{\Sigma}(G) \geq F
$$

\section{Backward}

Let $F, G \in \mathcal{C}\left(\mathbb{R}^{2}, \mathbb{R}\right)$.

$$
\begin{gathered}
\text { As } \forall x \in \mathbb{R}^{2} \quad F(x) \leq \underline{\Sigma}(G)(x), \\
\text { then } F(x) \leq \wedge\left\{s \mid y \notin \varepsilon_{s} X(G, s)\right\}, \\
\text { so } \forall s \in \mathcal{T}, x \notin \varepsilon_{s} X(G, s) \Longrightarrow F(x) \leq s . \\
\text { We deduce } \varepsilon_{s}(X(G, s))^{c} \subseteq\{x \mid F(x) \leq s\} .
\end{gathered}
$$


Moreover, as we obtain the following relation on the complementaries:

$$
\{x \mid F(x) \leq s\}^{c} \subseteq \varepsilon_{s}(X(G, s))
$$

so

$$
\delta_{s}(\{x \mid F(x)>s\}) \subseteq X(G, s)
$$

Consequently, on the superior bounds we have the following :

$$
\underbrace{\vee\left\{s \mid x \in \delta_{s} Y(F, s)\right\}}_{\bar{\Delta}(F)(x)} \leq \underbrace{\vee\{s \mid x \in X(G, s)\}}_{G(x)}
$$

We finally deduce:

$$
\bar{\Delta}(F) \leq G
$$

\subsection{Adaptation of the definitions in $\mathbb{Z}$}

In numerical image processing, one deals with discrete functions. Thus, one has to make sure that the definitions and the properties that were given in the continuous case are still valid for discrete functions, that is, for any function $F$ from a compact support of $\mathbb{Z}^{2}$ to $\mathbb{Z}$. First, note that the operators sup and inf behave differently in $\mathbb{R}$ and in $\mathbb{Z}$ : if $n \in \mathbb{Z}$ then $\sup \{s \in \mathbb{R} \mid s<n\}=n$ and $\sup \{s \in \mathbb{Z} \mid s<n\}=n-1$. Consequently, the proof given in section 3.2 is not correct in $\mathbb{Z}$. So the definitions of $\underline{\Sigma}$ and $\bar{\Delta}$ have to be modified to fit in $\mathbb{Z}$.

Let $\left(\varepsilon_{s}, \delta_{s}\right)_{s \in \mathbb{Z}}$ be a family of adjunctions on $\mathbb{Z}^{2}$, if

$\underline{\Sigma}:\left\{\begin{array}{l}\mathcal{C}\left(\mathbb{Z}^{2}, \mathbb{Z}\right) \rightarrow \mathcal{C}\left(\mathbb{Z}^{2}, \mathbb{Z}\right) \\ F \mapsto \underline{\Sigma}(F): x \mapsto \wedge\left\{s \in \mathbb{Z} \mid x \notin \varepsilon_{s+1} Y(F, s)\right\}\end{array}\right.$

$\bar{\Delta}:\left\{\mathcal{C}\left(\mathbb{Z}^{2}, \mathbb{Z}\right) \rightarrow \mathcal{C}\left(\mathbb{Z}^{2}, \mathbb{Z}\right)\right.$

then

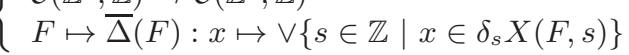

Theorem $(\underline{\Sigma}, \bar{\Delta})$ is an adjunction on $C\left(\mathbb{Z}^{2}, \mathbb{Z}\right)$.

Proof Let us prove that

$$
\forall F, G \in \mathcal{C}\left(\mathbb{Z}^{2}, \mathbb{Z}\right), \bar{\Delta}(F) \leq G \Longleftrightarrow F \leq \underline{\Sigma}(G)
$$

We may notice that the following definitions are equivalent:

- $\underline{\Sigma}(F)(x):\left\{\begin{array}{l}=\wedge\left\{s \in \mathbb{Z} \mid x \notin \varepsilon_{s+1} X(F-1, s)\right\} \\ =\wedge\left\{s \in \mathbb{Z} \mid x \notin \varepsilon_{s+1} Y(F, s)\right\} \\ =\wedge\left\{s \in \mathbb{Z} \mid x \notin \varepsilon_{s} Y(F-1, s)\right\}-1\end{array}\right.$

- $\bar{\Delta}(F)(x):\left\{\begin{array}{l}=\vee\left\{s \in \mathbb{Z} \mid x \in \delta_{s} Y(F+1, s)\right\} \\ =\vee\left\{s \in \mathbb{Z} \mid x \in \delta_{s} X(F, s)\right\}\end{array}\right.$

The proof below is very similar to the one using operators on $\mathbb{R}^{2}$. It aims at justifying the change of definitions of $\bar{\Delta}$ and $\underline{\Sigma}$ in order to keep the adjunction property true.

\section{Forward}

Let $F, G \in \mathcal{C}\left(\mathbb{Z}^{2}, \mathbb{Z}\right)$.

$$
\begin{gathered}
\text { As } \forall y \in \mathbb{Z}^{2}, \bar{\Delta}(F)(y) \leq G(y), \\
\text { then } \vee\left\{s \mid y \in \delta_{s} Y(F+1, s)\right\} \leq G(y), \\
\text { so } \forall s \in \mathbb{Z}, y \in \delta_{s} Y(F+1, s) \Longrightarrow s \leq G(y) .
\end{gathered}
$$


We deduce $Y(F+1, s) \subseteq \varepsilon_{s}(\{y \mid G(y) \geq s\})$,

and by adjunction $Y(F, s) \subseteq \varepsilon_{s}(\{y \mid G(y) \geq s\})$,

which means $Y(F+1, s) \subseteq \varepsilon_{s}(X(G, s))$.

Then, as we have the following relation on the complementaries:

$$
\varepsilon_{s}(X(G, s))^{c} \subseteq Y(F+1, s)^{c}
$$

Then, on the inferior bounds $\forall x$, it is successively true that

$$
\begin{gathered}
\wedge\left\{s \mid x \in \varepsilon_{s}(X(G, s))^{c}\right\} \geq \wedge\left\{s \mid x \in Y(F+1, s)^{c}\right\} \\
\wedge\left\{s \mid x \notin \varepsilon_{s}(X(G-1, s-1))\right\} \geq \wedge\{s \mid F(x)+1 \leq s\} \\
\wedge\left\{s+1 \mid x \notin \varepsilon_{s+1}(X(G-1, s))\right\} \geq \wedge\{s+1 \mid F(x) \leq s\} \\
\underbrace{\wedge\left\{s \mid x \notin \varepsilon_{s+1}(X(G-1, s))\right\}}_{\underline{\Sigma}(G)(x)}+1 \geq \underbrace{\wedge\{s \mid F(x) \leq s\}}_{F(x)}+1
\end{gathered}
$$

Eventually, we obtain:

$$
\underline{\Sigma}(G) \geq F
$$

\section{Backward}

Let $F, G \in \mathcal{C}\left(\mathbb{Z}^{2}, \mathbb{Z}\right)$.

$$
\begin{gathered}
\text { As } \forall x \in \mathbb{Z}^{2}, F(x) \leq \underline{\Sigma}(G)(x), \\
\text { then } F(x) \leq \wedge\left\{s \mid y \notin \varepsilon_{s+1} X(G-1, s)\right\}, \\
\text { so } \forall s \in \mathbb{Z}, x \notin \varepsilon_{s+1} X(G-1, s) \Longrightarrow F(x) \leq s .
\end{gathered}
$$

We deduce $\varepsilon_{s+1}(X(G-1, s))^{c} \subseteq\{x \mid F(x) \leq s\}$,

Consequently, we have the following relation on the complementaries:

$$
\begin{aligned}
& \{x \mid F(x) \leq s\}^{c} \subseteq \varepsilon_{s+1}(X(G-1, s)) \\
& \delta_{s+1}(\{x \mid F(x)>s\}) \subseteq X(G-1, s)
\end{aligned}
$$

Then, on the superior bounds, it is successively true that

$$
\begin{gathered}
\vee\left\{s \mid x \in \delta_{s+1} Y(F, s)\right\} \leq \vee\{s \mid x \in X(G-1, s)\} \\
\vee\left\{s \mid x \in \delta_{s+1} Y(F+1, s+1)\right\} \leq \vee\{s \mid G(x)-1 \geq s\} \\
\vee\left\{s-1 \mid x \in \delta_{s} Y(F+1, s)\right\} \leq \vee\{s-1 \mid G(x) \geq s\} \\
\underbrace{\vee\left\{s \mid x \in \delta_{s} Y(F+1, s)\right\}}_{\bar{\Delta}(F)(x)}-1 \leq \underbrace{\vee\{s \mid G(x) \geq s\}}_{G(x)}-1
\end{gathered}
$$

We eventually obtain:

$$
\bar{\Delta}(F) \leq G
$$

\section{EXPERIMENTAL APPLICATIONS}

We first show how adaptive operators work on numerical images for various structuring elements. Then, we illustrate two applications of adaptive closings and openings. Finally we present an idea of how to remove noises from images using adaptive morphology. 


\subsection{Effect of diverse semi-flat dilations and erosions}

The classical grey-tone image "Lena" is used as reference image for all the transformations. The dynamic of the image were reduced to 128 (instead of 256) for implementation reasons.

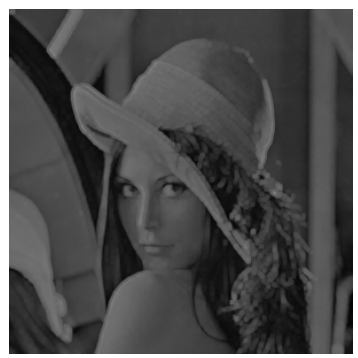

In Fig.6, a square structuring element of size $11-(2 \operatorname{floor}((s-1) / 20)+1))$, for $s \leq 101$ (where $s$ is the grey level) were used. The effect of the associated semi-flat closing is presented: only the zones of low grey levels are closed ; zones of highest grey-levels are left unchanged.

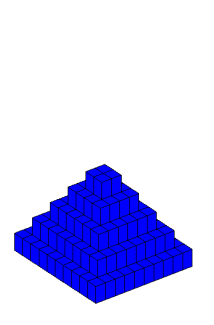

(a) Structuring element

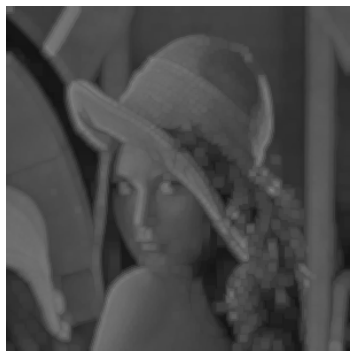

(b) semi-flat dilation

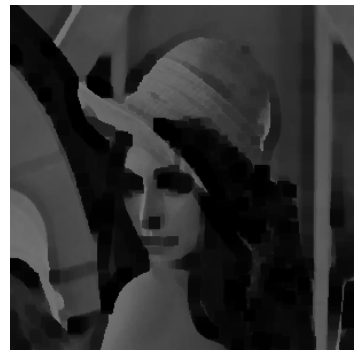

(c) semi-flat erosion

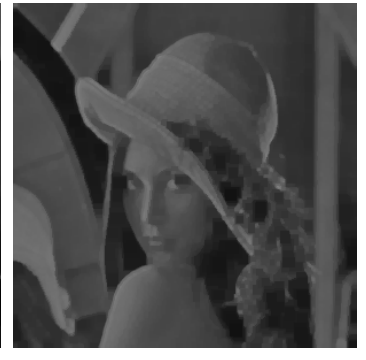

(d) semi-flat closing

Figure 6. Example of semi-flat operators in the case of a non decreasing family of set operators.

In, the example of Fig.??, the size of the structuring element is now increasing with the intensity: zones of highest grey-levels are dilated, eroded or closed.

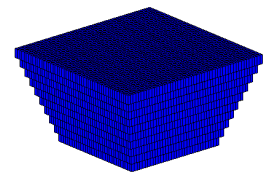

(a) Structuring element

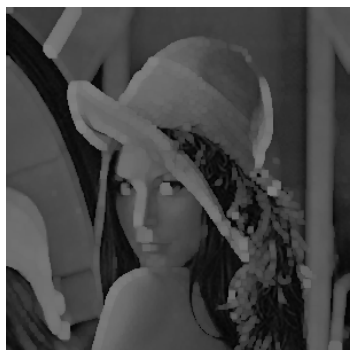

(b) adaptive dilation

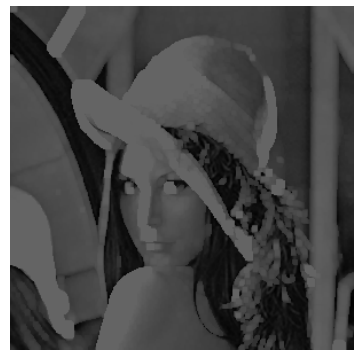

(c) adaptive closing

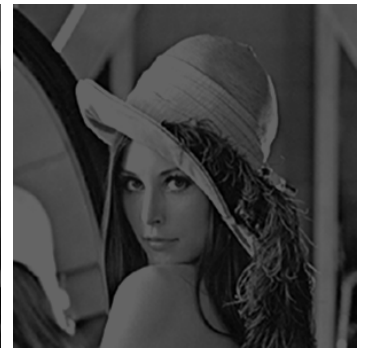

(d) adaptive erosion

Figure 7. The same as figure 6 using a "reverse" structuring element.

\subsection{Connection of thin lines by semi-flat dilation}

The fig. 8 illustrates the behavior of a semi-flat dilation of type 1 (the type as the one presented in figure 6 ) and its use for reconnecting thin contours lines. Here the goal is to extract the roads in the air image. The semi-flat operator preserves high intensities while dilating points of lowest intensity. The result is that crest lines of the original image are preserved while holes (due to noise, umbra or vegetation) are filled. 


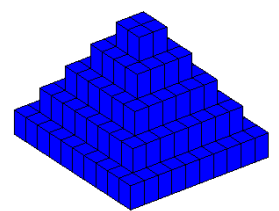

(a) Structuring element

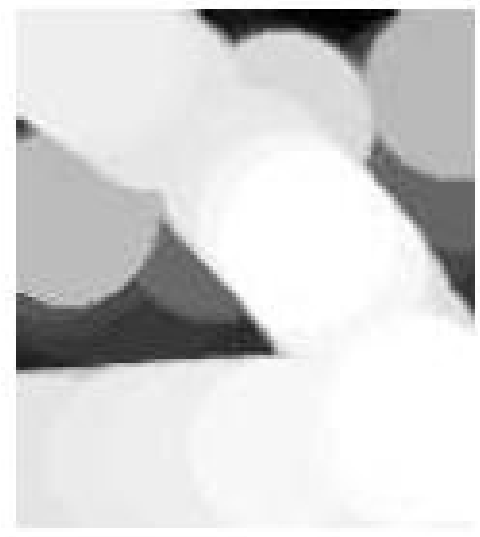

(c) Result of a flat dilation

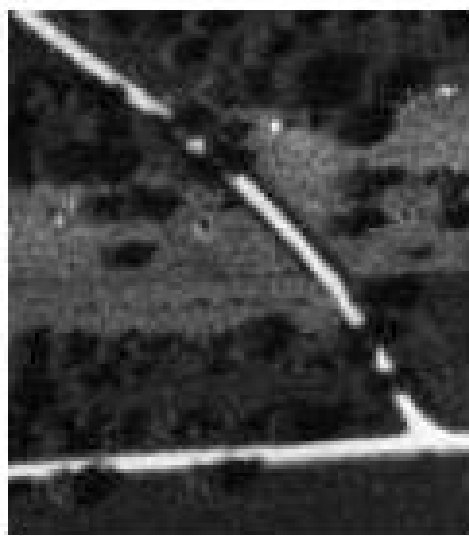

(b) Original image

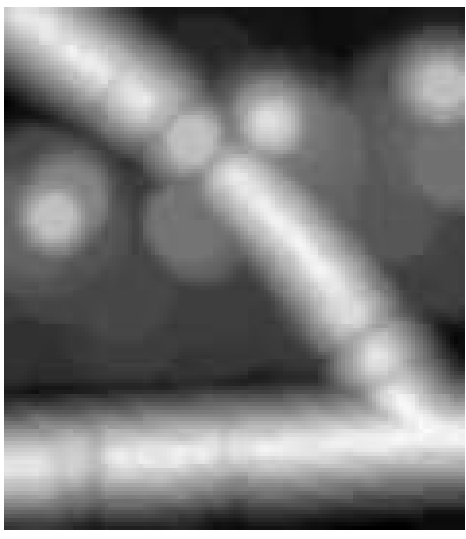

(d) Result of the semi-flat dilation

Figure 8. Connection of thin lines by semi-flat dilation

\subsection{Image simplification by semi-flat filtering}

In the example of figure 9, a color image is processed. As classically in mathematical morphology, the function values of the color map are ordered. The ordering is shown as a grey-tone image. In this example, the ordering is defined as a distance map, the colors set of reference containing the colors of the flowers. Semi-flat filters are then applied on the color image. The structuring element is of type 2 (see figure 7). Thus, the zones of high distance from the reference are hardly filtered while the flowers (as every point of low distance) are preserved. Here openings and closings have been sequentially applied on the image in order to process white and dark zones as well.

\section{CONCLUSION}

In this paper, semi-flat morphological transformations of grey-scale images were proposed that are valid when applying any kind of set operators on the level sets of the image. One important result is that couples of operators defining adjunctions can be defined using the proposed paradigm. This guarantees that idempotent operators can be obtained if the family of set operators is chosen in this perspective.

Semi-flat transformations are intensity-adaptive transformations: the image shapes are processed according to their grey-levels. Usefulness of such kind of transformations were illustrated by some examples. Other applications could involve image denoising (for radar images for instance as the local noise intensity is linked to 


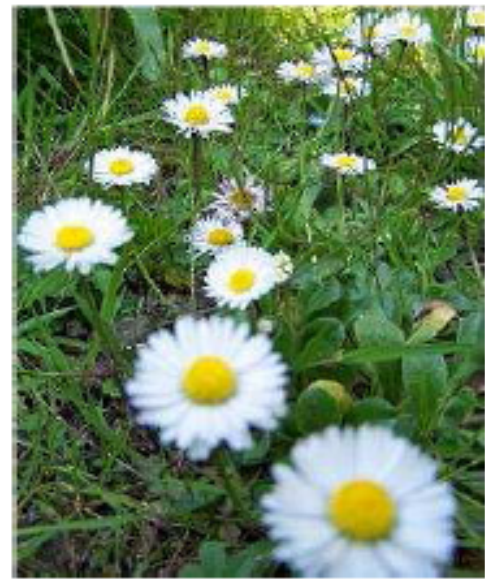

(a) Original image

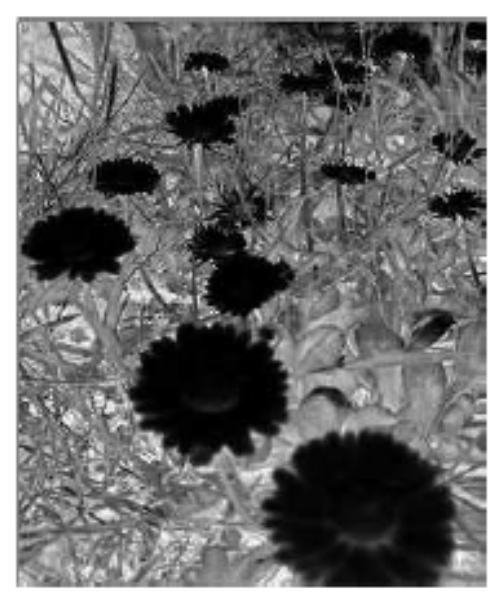

(b) Distance map (the reference set is a daisy)

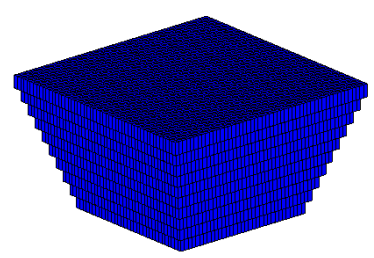

(c) Structuring element

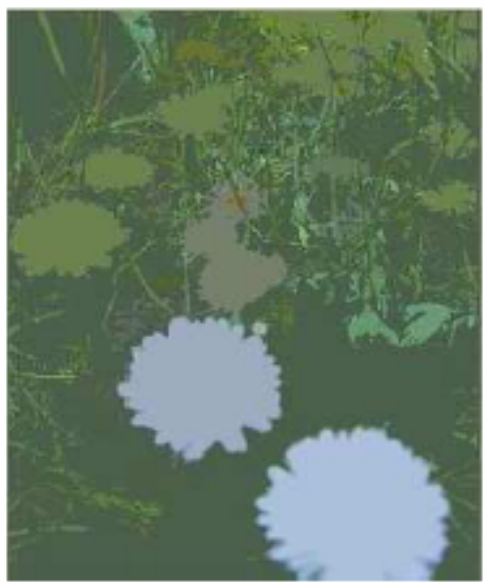

(d) Standard filtering (defined using the flat extension paradigm)

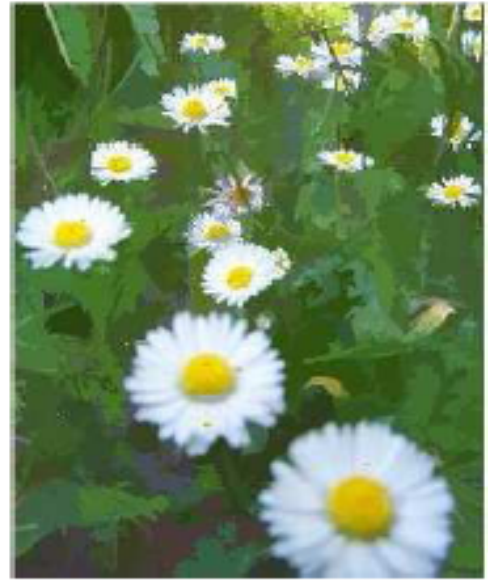

(e) Adaptive filtering (defined using the proposed semi-flat extension paradigm)

Figure 9. Simplification of a color image by adaptive filtering of a distance map.

the local luminance in the image), image simplification (if the intensity 0 is redefined as a reference luminance) and contrast enhancement (see intensity-adaptive filters as a variant of the shock filters).

One key point of the work on that subject will be the implementation. As suggested in, ${ }^{12}$ numerical implementations of PDE governing multi-scale adaptive dilations and erosions could be useful.

\section{REFERENCES}

[1] F. Guichard, J.-M. Morel, and R. Ryan, Contrast invariant image analysis and PDEs.

[2] R. Brockett and P. Maragos, "Evolution equations for continuous-scale morphological filtering," IEEE Transactions on Signal Processing 42, 1994.

[3] F. Guichard, P. Maragos, and J.-M. Morel, "Partial differential equations for morphological operators," Tribute to Georges Matheron, Springer-Verlag, 2005.

[4] M. Crandall, Viscosity Solutions: A Primer, University of California, year $=$.

[5] J. Serra, Image Analysis and Mathematical Morphology, vol. 2: Theoretical Advances, Acad. Press, year =. $[6]$ 
[7] P. Maragos and F. Meyer, "Nonlinear pdes and numerical algorithms for modeling levelings and reconstruction filters," Scale-Space'99, Springer-Verlag, 1999.

[8] P. Maragos and F. Meyer, "Morphological scale-space representation with levelings," Scale-Space'99, Springer-Verlag, 1999.

[9] P. Dokladal, Méthodes à ensembles de niveau en analyse d'images. PhD thesis, ENSTA, 2007.

[10] B. Tremblais, B. Augereau, and M. Leard, "Shock filters and mathematical morphology," tech. rep., Université de Poitiers, 2001.

[11] J.-F. Aujol, "Traitement d'images par approches variationnelles et équations aux dérivées partielles," tech. rep., CMLA, ENS Cachan, CNRS, PRES UniverSud, 2005.

[12] P. Maragos and C. Vachier, "A pde formulation for viscous morphological operators with extensions to intensity-adaptive operators," Proc. of IEEE International Conference on Image Processing, 2008. 